\title{
Exponential increase in postprandial blood-glucose exposure with increasing carbohydrate loads using a linear carbohydrate-to-insulin ratio
}

\author{
K J Marran, ${ }^{1}$ MB BCh, FCPaeds (SA), MMed Paeds, Cert Paeds Endo (SA); B Davey, ${ }^{2}$ BA (Hons) (Biokinetics), Dip (Diabetes) (Cardiff); \\ A Lang, ${ }^{2}$ BSc (Dietetics), PGDip (Dietetics); D G Segal, ${ }^{1}$ MB BCh, FAAP (USA), FACE
}

${ }^{1}$ Department of Paediatric Endocrinology, Charlotte Maxeke Academic Hospital, Johannesburg, South Africa

${ }^{2}$ Donald Gordon Medical Centre, University of the Witwatersrand, Johannesburg, South Africa

Corresponding author: KJ Marran (kerry@endo.co.za)

\begin{abstract}
Background. Postprandial glucose excursions contribute significantly to average blood glucose, glycaemic variability and cardiovascular risk. Carbohydrate counting is a method of insulin dosing that balances carbohydrate load to insulin dose using a fixed ratio. Many patients and current insulin pumps calculate insulin delivery for meals based on a linear carbohydrate-to-insulin relationship. It is our hypothesis that a non-linear relationship exists between the amounts of carbohydrate consumed and the insulin required to cover it.

Aim. To document blood glucose exposure in response to increasing carbohydrate loads on fixed carbohydrate-to-insulin ratios. Methods. Five type 1 diabetic subjects receiving insulin pump therapy with good control were recruited. Morning basal rates and carbohydrateto-insulin ratios were optimised. A Medtronic glucose sensor was used for 5 days to collect data for area-under-the-curve (AUC) analysis, during which standardised meals of increasing carbohydrate loads were consumed.

Results. Increasing carbohydrate loads using a fixed carbohydrate-to-insulin ratio resulted in increasing glucose AUC. The relationship was found to be exponential rather than linear. Late postprandial hypoglycaemia followed carbohydrate loads of $>60 \mathrm{~g}$ and this was often followed by rebound hyperglycaemia that lasted $>6$ hours.

Conclusion. A non-linear relationship exists between carbohydrates consumed and the insulin required to cover them. This has implications for control of postprandial blood sugars, especially when consuming large carbohydrate loads. Further studies are required to look at the optimal ratios, duration and type of insulin boluses required to cover increasing carbohydrate loads.
\end{abstract}

S Afr Med J 2013;103(7):461-463. DOI:10.7196/SAMJ.6382

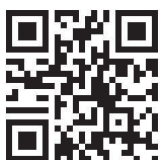

Type 1 diabetes is a complex disease requiring insulin administration in conjunction with an individual meal plan that takes into consideration social, economic and logistical factors to achieve optimal diabetes control. Short-term goals are to ensure normal growth and development, while minimising complications such as hyper- and hypoglycaemia. Long-term goals are to achieve blood sugars as close to the normal range as possible, to prevent or delay devastating long-term complications.

The diabetes control and complication trial (DCCT) proved that lowering haemoglobin $\mathrm{AlC}(\mathrm{HbAlc})$ through intensive management effectively delayed the onset and slowed the progression of diabetic retinopathy, nephropathy and neuropathy. ${ }^{[1]}$ Further analysis of DCCT trial data and other evidence suggests that glycaemic variability contributes to diabetes complications independently of the HbA1c. ${ }^{[2,3]}$

The contribution of postprandial hyperglycaemia to the average blood glucose level, measured by HbAlc, increases in an inverse relationship to HbAlc. ${ }^{[4]}$ Reducing postprandial hyperglycaemia is essential to achieving the published target of $<7 \%$ HbAlc. ${ }^{[4]}$ Furthermore, a rise in postprandial glucose (PPBG) is an independent risk factor for cardiovascular events. ${ }^{[5]}$ In well-controlled patients, it is a stronger predictor of cardiovascular disease than elevations of fasting blood glucose. ${ }^{[6]}$ Thus, PPBG concentrations, which contribute significantly to average blood-glucose measurements, may well be an independent risk factor for other diabetic complications and need to be targeted independently of the mean blood sugar, as measured by the HbAlc.
Dietary management in diabetes aims to provide the recommended daily intake of energy and macro- and micronutrients, while minimising glucose exposure and fluctuations. Dietary carbohydrate is the primary determinant of meal-related blood-glucose excursions and is dependent on the glycaemic index (GI) and the overall load of carbohydrate consumed. ${ }^{[7]}$ Patients with diabetes have a higher and more prolonged postprandial glucose excursion due to abnormalities in insulin, glucagon and amylin secretion, hepatic and peripheral glucose uptake, and suppression of hepatic glucose production. ${ }^{[7]} \mathrm{A}$ tighter match between insulin action and carbohydrate delivery will improve blood-glucose control while reducing the occurrence of both hyper- and hypoglycaemia.

Carbohydrate counting is a method that matches carbohydrate intake with insulin delivery and has been shown to offer an advantage in terms of greater reduction in HbAlc than standard meal planning. ${ }^{[8]}$ The American Diabetes Association currently recommends that prandial insulin dosing is based on the total carbohydrate content of the meal, but consideration should be given to the GI and glycaemic load of the meal. ${ }^{[9]}$

Following the DCCT trial, intensified insulin therapy is the gold standard for managing diabetes and forms the backbone of most modern insulin regimens. The majority of modern insulin regimens and bolus advisors use some form of carbohydrate counting and a linear algorithm to match bolus mealtime insulin to the amount of carbohydrate eaten.

Previous euglycaemic clamp studies found a linear relationship between the insulin infused and carbohydrate load. ${ }^{[10]}$ However, significant differences exist between the pharmacokinetic and pharmacodynamic properties of subcutaneously injected bolus insulin and continuous intravenous insulin infusions. 
Based on our clinical experience, we hypothesised that a non-linear relationship exists between the amount of carbohydrate consumed and the insulin ratio required to cover it when using pre-meal subcutaneous bolus insulin. No previous studies have been done using subcutaneously injected insulin to examine the relationship between the amount of carbohydrate consumed and the amount of insulin required to maintain euglycaemia.

According to our hypothesis, as the carbohydrate load increases, the amount of insulin required will increase exponentially rather than linearly. To test this we used real-time continuous glucose monitoring to assess blood-glucose exposure, using fixed carbohydrate ratios with the delivery of increasing carbohydrate loads.

\section{Methods}

\section{Study design and subjects}

We performed an analytical observational study on 5 type 1 diabetic adolescents and young adults receiving insulin pump therapy with good control and stable carbohydrate-to-insulin ratios attending the Centre for Diabetes and Endocrinology at the Donald Gordon Medical Centre, Johannesburg, South Africa. Average participant age was 21.4 years (range 14 - 30), with a mean duration of diabetes of 8.4 years $(4-20)$. The mean $\mathrm{HbAlc}$ of the participants was $7.1 \%$ (6.8 - 7.4\%).

Participants' basal rates and carbohydrate ratios were optimised prior to the study. A Medtronic continuous glucose monitoring system (CGMS) (Medtronic Minimed, USA) was inserted and calibrated for each participant on the first day. Participants continued regular selfmonitoring blood glucose checks and calibration checks.

For 5 consecutive days the participants ate, in random order, a preprepared breakfast of $30 \mathrm{~g}, 60 \mathrm{~g}, 90 \mathrm{~g}, 120 \mathrm{~g}$ and $150 \mathrm{~g}$ carbohydrate (2C, 4C, 6C, 8C, 10C meals, respectively) (Table 1). The content was calculated and prepared by a registered dietician. The participants ate breakfast at the same time on each study day and gave themselves a bolus of insulin 15 minutes before eating, according to their predetermined carbohydrate-to-insulin ratios. At the end of the 5-day period, the CGMS was removed and the data downloaded using Medtronic Solutions software. Each participant performed the 5-day study procedure twice so that inter- and intra-patient variability could be compared.

The glucose level was captured every 5 minutes from baseline to the peak of glucose absorption and back to baseline (Fig. 1). The area under the curve (AUC) was calculated for each meal for each participant, representing the 'incremental glucose area' or postprandial glucose exposure. The AUC for blood-glucose excursion was compared for the 5 different meals. The maximal change in glucose for each meal was documented, as was the time taken to reach this glucose level and the time taken for glucose level to return to baseline.

\section{Statistical analysis}

A straight line was fitted to the log data using an analysis of covariance, allowing for possible differences between the 5 individual participants. $P$-values were determined to assess the significance of the fit of the line. Predicted values were obtained from the fitted model. A one-way randomised blocks design was used to determine the significance of the maximal change in glucose for each meal, the time to maximum glucose and the time taken for glucose to return to baseline.

\section{Results}

There was a significant increase $(p<0.0002)$ in the peak glucose and time to return to baseline $(p<0.002)$ as carbohydrate loads increased, without a significant increase in time to peak levels. The AUC for each participant for each meal increased with increasing carbohydrate load, with an exponential rather than linear trend (Fig. 2) - confirmed by

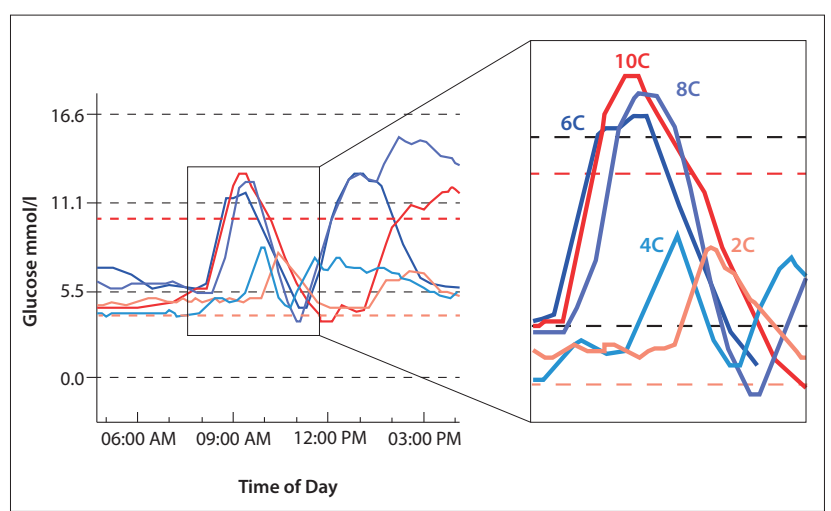

Fig. 1. Blood glucose levels for a single participant's 5-day study period. Each line represents a different carbohydrate meal $(2 \mathrm{C}, 4 \mathrm{C}, 6 \mathrm{C}, 8 \mathrm{C}, 10 \mathrm{C})$.

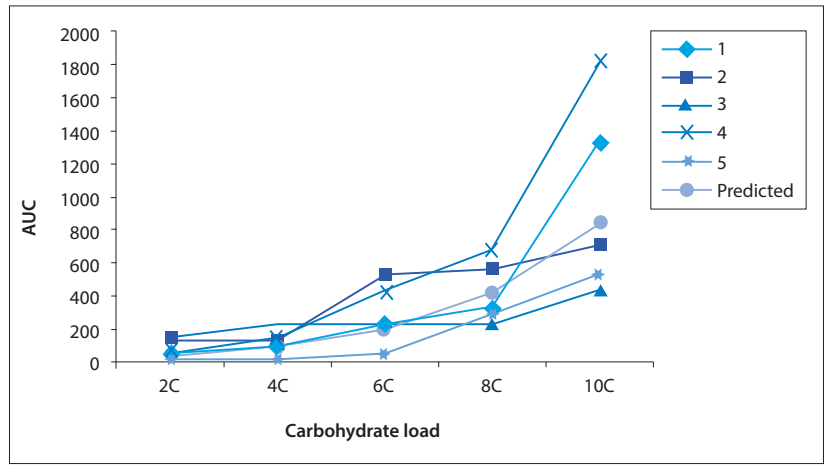

Fig. 2. Area under the curve (AUC) for each meal (carbohydrate load) for participants $1-5$.

Table 1. Nutritional content of each meal

\begin{tabular}{|c|c|c|c|c|c|c|}
\hline $\begin{array}{l}\text { Carbohydrate meals } \\
n\end{array}$ & $\begin{array}{l}\text { Carbohydrate } \\
\text { g }\end{array}$ & $\begin{array}{l}\text { Nutren Diabetes* } \\
\mathrm{ml}(\mathrm{g})\end{array}$ & $\begin{array}{l}\text { Fruit squares }{ }^{\dagger} \\
n(\mathrm{~g})\end{array}$ & $\begin{array}{l}\text { Marie biscuits }{ }^{\ddagger} \\
n(\mathrm{~g})\end{array}$ & $\begin{array}{l}\text { Protein content } \\
\mathrm{g}\end{array}$ & $\begin{array}{l}\text { Fat content } \\
\mathrm{g}\end{array}$ \\
\hline 2 & 30 & $75(15)$ & $5(10)$ & $1(5)$ & 1.55 & 1.04 \\
\hline 4 & 60 & $150(30)$ & $10(20)$ & $2(10)$ & 3.30 & 2.08 \\
\hline 6 & 90 & $225(45)$ & $15(30)$ & $3(15)$ & 4.95 & 3.12 \\
\hline 8 & 120 & $300(60)$ & $20(40)$ & $4(20)$ & 6.60 & 4.16 \\
\hline 10 & 150 & $375(75)$ & $25(50)$ & $5(25)$ & 8.25 & 5.20 \\
\hline Carbohydrate, \% & - & 50 & 33 & 17 & - & - \\
\hline
\end{tabular}


the significant linear relationship between the natural logarithm of the AUC and the carbohydrate load for each participant, as well as for the average of all 5 participants $(p<0.0001)$. An analysis of covariance did not reveal any significant differences between the participants.

The $2 \mathrm{C}$ and $4 \mathrm{C}$ meals showed a delay in time from eating to the start of the glucose rise and a smaller AUC. The $6 \mathrm{C}, 8 \mathrm{C}$ and $10 \mathrm{C}$ meals showed an earlier rise in blood glucose. There was an increase in time to maximum glucose from baseline, a greater peak and delayed return to baseline with these larger carbohydrate loads.

Following the higher glucose peak of the larger carbohydrate meals (6C, $8 \mathrm{C}$ and $10 \mathrm{C}$ meals; $>60$ g carbohydrate), the glucose level frequently dropped below baseline and often fell to the hypoglycaemic range $(<3.5 \mathrm{mmol} / \mathrm{l})$. This drop in glucose level, which was untreated in the study participants, rebounded and remained high for up to 6 hours. This high, followed by a low, followed by a high, shows a pattern of increasing glycaemic variability.

\section{Discussion}

Our analysis convincingly demonstrates that the AUC increases exponentially, and not linearly, with increasing carbohydrate load over the range of carbohydrate loads tested. This is the first study to document that, when using subcutaneous insulin, the linear algorithms used to calculate carbohydrate-to-insulin ratios may contribute to increased postprandial glucose excursions and variability when high carbohydrate loads are consumed.

This has implications for diabetics who use carbohydrate counting and carbohydrate-to-insulin ratios based on a linear algorithm to calculate pre-meal insulin doses. All 'smart' insulin pumps and 'bolus advisors' base their calculations on this linear algorithm.

Subcutaneously injected insulin has different pharmacokinetic properties to intravenously injected insulin with regard to onset of action, peak action and duration of action. As a result, studies that use intravenous insulin to derive the linear algorithm cannot be extrapolated to subcutaneously injected insulin.

The increase in postprandial blood sugars with the 6C - 10C meals is clinically significant and would contribute to higher $\mathrm{HbA1c}$ levels and cardiovascular risk. ${ }^{[4,5]}$ Higher postprandial glucose excursions with subsequent falls in blood glucose into the hypoglycaemic range and the rebound hyperglycaemia increase glucose variability. Both an increase in HbAlc and an increase in glucose variability have been shown to contribute to an increased risk of developing diabetic complications. ${ }^{[1-3]}$

The delayed hypoglycaemia following the $6 \mathrm{C}-10 \mathrm{C}$ meals has significant implications for management of these postprandial glucose excursions. Extrapolating from the exponential relationship, an exponentially larger dose of insulin would be required to prevent the postprandial glucose rise. However, owing to the pharmacokinetic properties of subcutaneous insulin and the finding of delayed hypoglycaemia, it is possible to deduce that this would only lead to a greater frequency and severity of late postprandial hypoglycaemia.

There are a few possible management options for controlling the postprandial hyperglycaemia observed with larger carbohydrate meals:

- Limit carbohydrate intake at each meal to a maximum of $4 \mathrm{C}$. We have shown that the glucose excursion after ingesting up to $4 \mathrm{C}(60 \mathrm{~g}$ carbohydrate) can be limited by preprandial subcutaneous insulin using fixed insulin-to-carbohydrate ratios and that the slope of the curve is more linear in this range. Low carbohydrate diets have proven efficacy in reducing postprandial glucose levels, $\mathrm{HbAlc}$ and frequency of hypoglycaemic episodes. ${ }^{[1]}$ Low carbohydrate diets limit total daily intake to $\leq 70$ - $90 \mathrm{~g}$ carbohydrate. These strict targets are difficult to maintain over the long term and, based on our data, less stringent carbohydrate targeting could be recommended, provided that meals $>4 \mathrm{C}$ are avoided.
- Administer prandial insulin 20 - 30 min before the meal to ensure sufficient insulin to meet and store the incoming carbohydrates.

- Change the pharmacokinetics of the injected insulin to accelerate its absorption or change its delivery profile. The newly developed heating patch, InsuPatch, has significant effects on the pharmacokinetics of insulin analogues and PPBG. ${ }^{[12]}$ Patients receiving insulin pump therapy can change the duration over which their pre-meal insulin is injected. Insulin can be injected using a 'square wave' bolus, where the total amount of insulin is infused over a set time period, or a 'dual wave' bolus, where a percentage of the dose can be injected pre-meal and the remaining percentage infused over a set time period Both the percentage injected and the time period over which it is injected can be adjusted. ${ }^{[13]}$ Newer, faster-acting insulins are also being developed that hold promise for PPBG control.

- Recommend the consumption of lower GI carbohydrates, which have been shown to lower PPBG. ${ }^{[14]}$

This study is limited in that only 5 subjects were involved. Further studies are required to determine the optimal ratios of insulin, insulin delivery options and non-insulin-related strategies for managing PPBG. Our study's strength lies in showing the importance of insulin dose regulation by diabetics according to the carbohydrate loads consumed.

\section{Conclusion}

To prevent complications of diabetes, patients need to reduce their average blood sugars, as measured by $\mathrm{HbAlc}$, and their glycaemic variability to as close to the non-diabetic range as possible. PPBG levels contribute significantly to $\mathrm{HbAlc}$ and glycaemic variability, hence the importance of optimally limiting post-meal glucose excursions. Dietary carbohydrate is the primary determinant of PPBG excursions with preprandial bolus insulin primarily governing the excursion of postprandial glucose.

We have shown that the consumption of an increasing carbohydrate load leads to an exponential increase in postprandial glucose exposure when using a linear algorithm to determine the insulin-tocarbohydrate ratio after subcutaneous insulin injection, and increases the risk for delayed postprandial hyper- and hypoglycaemia.

\section{References}

1. The Diabetes Control and Complications Trial Research Group. The effect of intensive treatment of diabetes on the development and progression of long-term complications in insulin-dependent diabetes mellitus. N Engl J Med 1993;329(14):977-986. [http://dx.doi.org/10.1056/NEJM199309303291401] 2. Hirsch IB. Glycaemic variability: It's not just about AIC anymore! Diabetes Technol Ther 2005;7(5):780-783

3. Nalysnyk L, Hernandez-Medina M, Krishnarajah G. Glycaemic variability and complications in patient with diabetes mellitus: Evidence from a systematic review of the literature. Diabetes Obes Metab 2010;12(4):288-298. [http://dx.doi.org/10.1111/j.1463-1326.2009.01160.x]

4. Woerle HJ, Neumann C, Zschau S, et al. Impact of fasting and postprandial glycaemia on overall glycaemic control in type 2 diabetes. Importance of postprandial glycaemia to achieve target HbAlc levels. Diabetes Res Clin Pract 2007:77(2):280-285. [http.//dx doi org/10.1016/j diabres 2006 HbAl] levels. Diabetes Res Clin Pract 2007,77(2):280-285. [http://dx.doi.org/10.1016/j.diabres.2006.11.011] Cavalot F, Petrelli A, Traversa M, et al. Postprandial blood glucose is a stronger predictor of cardiovascular
events than fasting blood glucose in type 2 diabetes mellitus, particularly in women: Lessons from the San events than fasting blood glucose in type 2 diabetes mellitus, particularly in wo
Luigi Gonzaga Diabetes Study. I Clin Endocrinol Metab 2006;91(3):813-819. 6. Monnier L, Lapinski H, Colette C. Contributions of fasting and postprandial plasma glucose
increments to the overall diurnal hypoglycaemia of type 2 diabetic patients: variations with increasing increments to the overall diurnal hypoglycaemia of type 2 diabetic patients: variations with increasing
levels of $\mathrm{HbA}(1 \mathrm{c})$. Diabetes Care 2003;26(3):881-885. [http://dx.doi.org/10.2337/diacare.26.3.881] levels of $\mathrm{HbA}(1 \mathrm{c})$. Diabetes Care 2003;26(3):881-885. [http://dx.doi.org/10.2337/diacare.26.3.881]
American Diabetes Association. Postprandial blood glucose. Diabetes Care 2001;24(4):775-778.

7. American Diabetes Association. Postprandial blood glucose. Diabetes Care 2001;24(4):775-778.
8. Gillespie SJ, Kulkarni KD, Daly AE. Using carbohydrate counting in diabetes clinical practice. J Am Diet Assoc 1998;98(8):897-905. [http://dx.doi.org/10.1016/S0002-8223(98)00206-5]

Franz MJ, Bantle JP, Beebe CA, et al. Evidence-based nutrition principles and recommendations for the treatment and prevention of diabetes and related complications. Diabetes Care 2002;25(1):148-198. Kawamura T. The importance of carbohydrate counting in the treatment of children with diabetes. Pediatr Diabetes 2007;8:57-62. [http://dx.doi.org/10.1111/j.1399-5448.2007.00287.x]

Nielsen JV, Jonsson E, Ivarsson A. A low carbohydrate diet in type 1 diabetes: clinical experiencebrief report. Ups J Med Sci 2005;110(3):267-273. [http://dx.doi.org/10.3109/2000-1967-074]

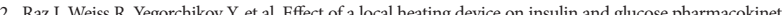
Raz I, Weiss R, Yegorchikov Y, et al. Effect of a local heating device on insulin and glucose pharmacokinetic profiles in an open-label, randomized, two-period, one-way crossover study in $\mathrm{P}$
using continuous subcutaneous insulin infusion. Clin Ther 2009:31(5):980-987.

O' pediatric patients with type 1 diabetes using insulin pump therapy: Impact of glycaemic index and pediatric patients with type 1 diabetes using insulin pump therapy: Impact of glycaemic index
prandial bolus type. Diabetes Care 2008;31(8):1491-1495. [http://dx.doi.org/10.2337/dc08-0306] prandial bolus type. Diabetes Care 2008;31(8):1491-1495. [http://dx.doi.org/10.2337/dc08-0306]
4. Ryan RL, King BR, Anderson DG, et al. Influence of and optimal insulin therapy for a low-glycaemi index meal in children with type 1 diabetes receiving intensive insulin therapy. Diabetes Care 2008;31(8):1485-1490. [http://dx.doi.org/10.2337/dc08-0331]

Accepted 15 October 2012. 